\title{
COMMENTARY
}

\section{Antiplatelet therapy: a double-edged sword in head injury?}

\author{
Christopher Beynon* and Oliver W Sakowitz \\ See related research by Fabbri et al., http://ccforum.com/content/17/2/R53
}

\begin{abstract}
Antiplatelet therapy for the treatment of cardiovascular diseases is common in the ageing population. Whether this therapy exacerbates brain injury after head trauma is an important, but unsettled, topic. In this issue of Critical Care, Fabbri and colleagues address the question of whether pre-injury intake of antiplatelet medication increases the risk profile of patients with posttraumatic intracranial lesions after head trauma. Antiplatelet medication, and in particular clopidogrel, increased the risk for haematoma progression, need for neurosurgical intervention and an unfavourable outcome. Clinicians should consider this increased risk profile in the treatment of respective patients. Since its introduction as an analgesic in 1897, aspirin has surprised the medical community more than once due to its versatile properties. Prevention of secondary brain damage through aspirin has been reported for ischaemic stroke and subarachnoid haemorrhage. In cases of acute traumatic haemorrhage after head injury, antiplatelet therapy's neuroprotective effects may be outweighed by the increased bleeding tendency.
\end{abstract}

This issue of Critical Care features an article on the outcome of patients with posttraumatic intracranial lesions after head trauma and a positive history of preinjury antiplatelet medication intake by Andrea Fabbri and colleagues from the Italian Society of Emergency Medicine [1]. Due to the high prevalence of cardiovascular diseases, the results of this study are of high interest to a broad audience from primary care to emergency departments and specialized neurotraumatological centres. Within the last decades, traumatic brain

*Correspondence: christopher.beynon@med.uni-heidelberg.de Department of Neurosurgery, Heidelberg University Hospital, Im Neuenheimer Feld 400, 69120 Heidelberg, Germany injury (TBI) has increasingly been recognized not only as 'the leading cause of mortality among patients under 45 years of age', but also as a growing disease in the ageing population of industrialized countries. Antiplatelet therapy is common in this population and has been associated with increased morbidity and mortality after head injury, but the evidence has been based mainly on pathophysiologic rationales and retrospective analyses with limited patient numbers [2]. Nevertheless, in some centres the scarce evidence available has led to protocols involving transfusion of platelet concentrates and administration of haemostatic substances for TBI patients with pre-injury use of antiplatelet medication. But are TBI patients really at increased risk for an unfavourable outcome if they have been treated with antiplatelet agents prior to injury?

The present study by Fabbri and colleagues adds important information to this discussion. The authors have to be applauded for the high total number of analysed patients with pre-injury intake of antiplatelet medication and posttraumatic intracranial lesions after head trauma. The number of 537 subjects, enabled by the multicentric study design with 32 participating centres, exceeds the total number of a recent meta-analysis of available studies on this topic [3] and allows conclusive statistical analyses. In this study, pre-injury intake of antiplatelet medication doubled the risk of lesion progression on computed tomography scans within 24 hours after trauma. Moreover, the risk of an unfavourable outcome on the Glasgow Outcome Scale 6 months after injury was increased by $50 \%$ in patients with pre-injury antiplatelet therapy.

What consequences should be drawn by the findings of this study? Appealing to primary care physicians for a more rational use of antiplatelet medication? More likely, optimizing current prescription practices and patient compliance would lead to even more patients treated with antiplatelet agents [4]. The present study demonstrates that if head injury results in posttraumatic intracranial lesions, these patients are at an increased risk for haematoma progression and an unfavourable outcome. This corresponds well with findings of Major and Reed 
[5], who observed a mortality rate of $21 \%$ in a study on patients with aspirin intake and posttraumatic intracranial haemorrhage. Clinicians must be aware of this increased risk profile and should adapt thresholds for clinical observation and repeated cranial imaging studies for respective patients. Routine application of haemostatic substances or transfusion of platelet concentrates cannot be recommended on the basis of available evidence [2]: still there is equipoise, whether counteracting antiplatelet therapy in the acute phase after TBI should be undertaken or not.

Increased bleeding tendencies may cause haematoma enlargement with concomitant mass effect on surrounding brain tissue. In the present study, antiplatelet therapy with aspirin and clopidogrel increased the risk of haematoma enlargement as well as the need for neurosurgical intervention. On the other hand, a recent study on 839 severely injured blunt trauma patients revealed that pre-injury treatment with antiplatelet agents reduced the risk of lung dysfunction and multiorgan failure if packed red blood cells were transfused [6]. Patients with severe TBI were excluded from data analysis in this study, but experimental studies have attributed several neuroprotective properties to aspirin [7]. Antiplatelet therapy in aneurysmal subarachnoid haemorrhage is associated with a trend towards better outcome in patients, possibly due to a reduced incidence of delayed cerebral ischaemia [8]. Coagulopathy after TBI is a dynamic process involving increased bleeding tendency as well as hypercoagulability leading to cerebral microthrombosis [9]. Posttraumatic cerebral microthrombosis, largely generated by platelet activation [10], may cause secondary brain injury due to cerebral hypoperfusion and ischaemia. Theoretically, antiplatelet therapy may also exert neuroprotective effects regarding the cerebral microcirculation after TBI. Nevertheless, in light of the available data, and in particular from the present study, it has to be assumed that in the acute phase after trauma, neuroprotective effects of antiplatelet therapy on the microcirculation are outweighed if computed tomography reveals posttraumatic intracranial lesions in patients. Further studies on the role of antiplatelet therapy in trauma and TBI are needed to optimize tomorrow's treatment protocols.

Abbreviations

$\mathrm{TBI}$, traumatic brain injury.

\section{Competing interests}

The authors declare that they have no competing interests.

Published: 23 April 2013

References

1. Fabbri A, Servadei F, Marchesini G, Bronzoni C, Montesi D, Arietta L, Societa Italiana di Medicina d'Emergenza Urgenza Study group: Antiplatelet therapy and the outcome of subjects with intracranial injury: the Italian SIMEU study. Crit Care 2013, 17:R53.

2. Beynon C, Hertle DN, Unterberg AW, Sakowitz OW: Clinical review: Traumatic brain injury in patients receiving antiplatelet medication. Crit Care 2012, $16: 228$.

3. Batchelor JS, Grayson A: A meta-analysis to determine the effect of preinjury antiplatelet agents on mortality in patients with blunt head trauma. Br J Neurosurg 2013, 27:12-18.

4. Russolillo A, Di Minno MND, Tufano A, Prisco D, Di Minno G: Filling the gap between science \& clinical practice: prevention of stroke recurrence. Thromb Res 2012, 129:3-8.

5. Major J, Reed MJ: A retrospective review of patients with head injury with coexistent anticoagulant and antiplatelet use admitted from a UK emergency department. Emerg Med J 2009, 26:871-876.

6. Harr JN, Moore EE, Johnson J, Chin TL, Wohlauer MV, Maier R, Cuschieri J, Sperry J, Banerjee A, Silliman CC, Sauaia A: Antiplatelet therapy is associated with decreased transfusion-associated risk of lung dysfunction, multiple organ failure, and mortality in trauma patients. Crit Care Med 2013, 41:399-404.

7. Crisanti P, Leon A, Lim DM, Omri B: Aspirin prevention of NMDA-induced neuronal death by direct protein kinase Czeta inhibition. J Neurochem 2005, 93:1587-1593.

8. Dorhout Mees SM, Van den Bergh WM, Algra A, Rinkel GJE: Antiplatelet therapy in aneurysmal subarachnoid hemorrhage. Stroke 2008 [Epub ahead of print].

9. Laroche M, Kutcher ME, Huang MC, Cohen MJ, Manley GT: Coagulopathy after traumatic brain injury. Neurosurgery 2012, 70:1334-1345.

10. Schwarzmaier SM, Kim S-W, Trabold R, Plesnila N: Temporal profile of thrombogenesis in the cerebral microcirculation after traumatic brain injury in mice. J Neurotrauma 2010, 27:121-130.

doi:10.1186/cc12597

Cite this article as: Beynon C, Sakowitz OW: Antiplatelet therapy: a doubleedged sword in head injury? Critical Care 2013, 17:135. 